\title{
COVID-19 and restrictive lung disease: A deadly combo to trip off the fine balance
}

\author{
Kamal Kant Sahu', Ajay Kumar Mishra', Kevin Martin², Iryna Chastain ${ }^{1}$ \\ ${ }^{1}$ Department of Internal Medicine, Saint Vincent Hospital, Worcester, MA; ${ }^{2}$ Division of Pulmonary and Critical Care, \\ Department of Internal Medicine, Worcester, MA, USA
}

\begin{abstract}
Coronavirus Disease (COVID-19) pandemic has so far led to innumerable deaths worldwide. The risk factors so far that have been most studied as poor prognostic factors are old age, individuals with multiple comorbidities and immunocompromised patients. Amongst the chronic lung diseases, most patients with COVID-19 reported so far had asthma, chronic obstructive pulmonary disease (COPD), and interstitial lung disease. Herein, we discuss the significance of restrictive lung disease during the COVID-19 pandemic as a potential risk factor via an example of a patient with kyphoscoliosis who succumbed to death due to COVID-19 pneumonia.
\end{abstract}

Correspondence: Kamal Kant Sahu, MD Medicine, Department of Internal Medicine, Saint Vincent Hospital, Worcester, MA 01608, USA. E-mail: drkksahu85@gmail.com

Key words: COVID-19; virus; lung; kyphoscoliosis.

Contributions: All the authors played a significant role in the paper. All the authors have read and approved the final version of the manuscript and agreed to be accountable for all aspects of the work.

Conflict of interest: The Authors have no conflict of interest to declare.

Ethical statement: No ethical committee approval was required for this case report by the Department, because this article does not contain any studies with human participants or animals. Informed consent was obtained from the patient included in this study.

Consent for publication: The patient gave his written consent to use his personal data for the publication of this case report and any accompanying images.

Received for publication: 27 April 2020.

Accepted for publication: 18 June 2020.

${ }^{\circ}$ Copyright: the Author(s), 2020

Licensee PAGEPress, Italy

Monaldi Archives for Chest Disease 2020; 90:1346

doi: 10.4081/monaldi.2020.1346

This article is distributed under the terms of the Creative Commons Attribution Noncommercial License (by-nc 4.0) which permits any noncommercial use, distribution, and reproduction in any medium, provided the original author(s) and source are credited.

\section{Case Report}

A 57-year-old non-smoker, with a history of severe kyphoscoliosis associated with the severe restrictive defect, hypertension, hyperlipidemia, presented with chief complaints of fever, nonproductive cough, and worsening shortness of breath of 2 days duration (Figure 1A).

On arrival his vitals were blood pressure 123/56 $\mathrm{mmHg}$, pulse rate 88 per min, respiratory rate 36 per min, temperature $101.3 \mathrm{~F}$, oxygen saturation $86 \%$ on 6 liters nasal cannula. He continued to become more tachypneic, with labored breathing and use of accessory respiratory muscles as well as paradoxical abdominal breathing. Soon he was intubated because of increased work of breathing, respiratory fatigue, drowsiness, and hypoxia despite being on 15 liters nonrebreather in addition to a high-flow cannula with $100 \% \mathrm{FIO}_{2}$. His initial blood gas showed a $\mathrm{Ph}$ of 7.289 with a $\mathrm{PCO}_{2}$ of 68.2 $\mathrm{mmHg}, \mathrm{PO}_{2}$ of $53.4 \mathrm{mmHg}, \mathrm{O}_{2}$ saturation of $81.8 \%$. His labs were notable for leukocytosis of 14,700 cells $/ \mathrm{mcL}$, neutrophilia of $88 \%$, lymphocytes $1300 / \mathrm{mcL}$, creatinine of 6.06 , anion gap $19 \mathrm{mEq} / \mathrm{L}$, bicarbonate $32 \mathrm{mmol} / \mathrm{L}$, glucose of 320 milligrams per deciliter, calcium $7.9 \mathrm{mg} / \mathrm{dL}$, albumin of $2.4 \mathrm{~g} / \mathrm{dL}$, lactate $1.6 \mathrm{mmol} / \mathrm{L}$, serum ferritin $854 \mathrm{ng} / \mathrm{mL}$, LDH 847 units per liter, and CRP $321 \mathrm{mg} / \mathrm{L}$.

His Chest CT without contrast revealed multifocal predominantly ground glass and peripherally based opacities with additional confluent consolidation of the right base (Figure 1B). His chest X-ray showed increased opacity in the right mid to lower Lung (Figure 1C). The COVID-19 PCR test from the nasopharyngeal swab came back as positive. His EKG showed classical features of dextrocardia due to the anomaly of thoracic bone architecture (Figure 2). Treatment with hydroxychloroquine, azithromycin, and ceftriaxone was initiated. Unfortunately, his oxygen requirement kept increasing $\left(\mathrm{FiO}_{2} 100 \%\right.$, PEEP of $16 \mathrm{~cm}$ $\mathrm{H} 2 \mathrm{O}$, Peak pressure of $46 \mathrm{~cm} \mathrm{H}_{2} \mathrm{O}$, Plateau pressure of $43 \mathrm{~cm}$ $\mathrm{H}_{2} \mathrm{O}$ ), with neuromuscular blockade and triple vasopressor support (epinephrine, norepinephrine, and vasopressin). He succumbed to illness on the $5^{\text {th }}$ day of hospital stay.

\section{Discussion}

This case was our unique experience as we are seeing more COPD or interstitial lung disease as a factor for compromised lung function. The common CT chest findings of COVID-19 are bilateral patchy shadows or ground-glass opacities in the bilateral lungs $[1,2]$. We reviewed a few of the major studies reported from China and did not find any description of the restrictive lung disorders [35]. Almost all studies have quoted chronic lung disease as one of the 
important risk factors. However, most of them have not specified the type of lung disease they suffer from. So far, we have evidence of smoking and COPD as risk factors mentioned in these studies. But do we have any potentials significant difference of mortality, mechanical ventilation, or intensive care requirement amongst different types of chronic lung disorders? This is very much possible that most of the studies so far are not focusing on the thoracic anatomical anomalies as risk factors compromising lung functions and thereby impacting COVID-19 pneumonia outcome.
In the index case, the plateau and peak pressures were high, and this could have been just because of the patient's thoracic cage abnormality. In the absence of esophageal pressure monitoring, it is difficult to definitively suggest whether poor compliance was because of the thoracic cage or the lungs. Disproportionately higher $\mathrm{PCO}_{2}$ in acute on chronic respiratory acidosis, as compared to hypoxemia, also suggests the possibility that the kyphoscoliosis could be the primary culprit than the COVID pneumonia (which could be just a precipitating factor).
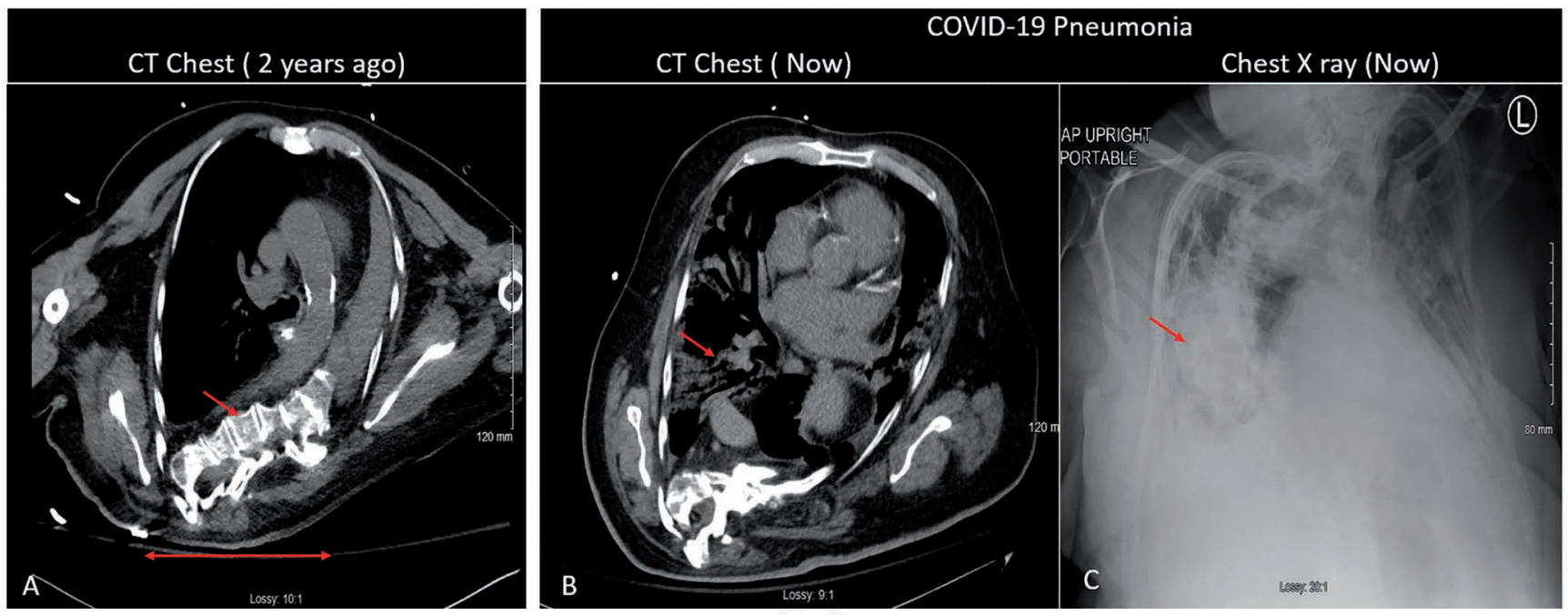

Figure 1. A) Baseline CT chest, 2 years ago (sagittal view) showing skewing of the thoracic framework with dextrocardia (mediastinal window) with significant loss of lung volume. B) CT chest (sagittal view) done during COVID-pneumonia showing consolidation, and breakdown. C) Chest X-ray showing opacities significantly involving the right lower and middle opacities.

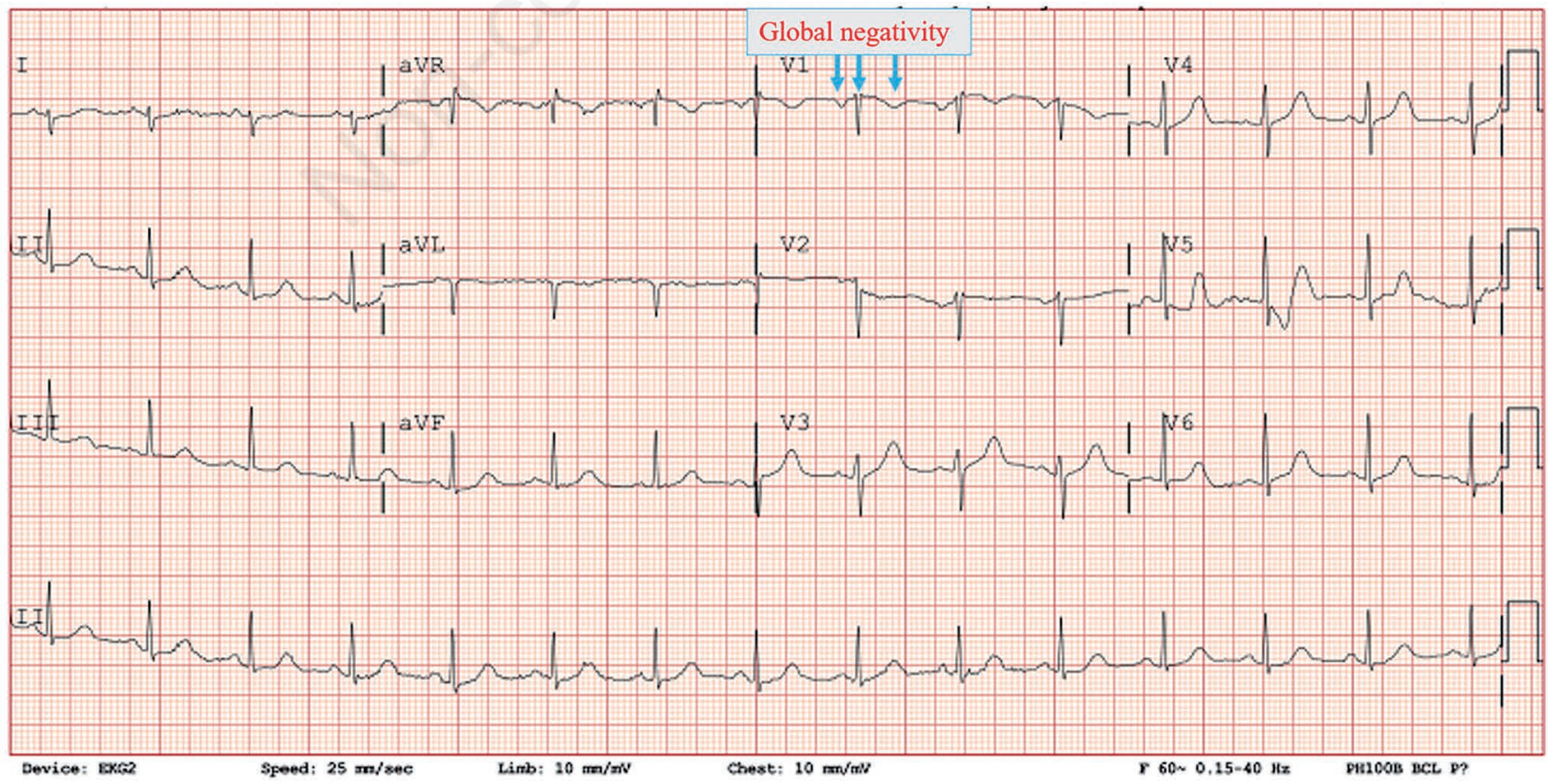

Figure 2. EKG showing inversion of all complexes, 'global negativity' (inverted P wave, negative QRS, inverted T wave). 
In addition, it is essential to endorse the importance of NIV trial in patients with COVID-19 in whom it can be safely and successfully used [6]. This is also supported by the evidence from previous studies on coronavirus outbreaks. The literature has conflicting reports with epidemiological data suggesting that NIV could promote nosocomial transmission. In contrast, human laboratory data suggests that it does not generate enough aerosols [7-9]. In a 20-patient series of SARS related pneumonia, timely use of NIV effectively prevented endotracheal intubation in $70 \%$ cases [10]. During the initial few weeks of the COVID-19 pandemic, there was a scarcity of appropriate protective equipment (PPEs) to the health care providers [11]. In addition, few studies also suggested the benefits of early intubation to prevent patient self-induced lung injury [12]. However, considering the potential benefit of avoiding intubation, ensuring a good interface fitting for NIV, and appropriate use of PPE, intensivists need to use clinical judgment if patients could be benefitted from noninvasive strategies without compromising the health of health care providers [13]. Phua et al. recently suggested intensive care management recommendations which discussed the feasibility and potential benefits of NIV use [9].

Another unique characteristic of COVID-19 pneumonia is the recognition of different respiratory phenotypes $[6,14,15]$. The special population of COVID-19 is often described as COVID-19's 'happy hypoxia' in which patients are presenting with oxygen saturation well below $90 \%$, but still without any respiratory discomfort or shortness of breath [6,15]. Gattinoni et al. described COVID-19 pneumonia disease spectrum in two primary "phenotypes", namely COVID-19 pneumonia, Type L, and COVID-19 pneumonia, Type $\mathrm{H}$ and proposed a different respiratory treatment for each of them [6].

Apart from the respiratory system, impact of COVID-19 on various other organ systems and their functioning are currently being studied by various basic science researchers and scholars [16]. Based on the available literature or proposed pathophysiological mechanisms, various newer diagnostic and therapeutic modalities are being studied for COVID-19.

\section{Conclusions}

Hence, our case is an attempt to sensitize the research bodies to look for the association between the other lung pathologies (in addition to usual COPD, ILD) like scoliosis, kyphosis, and sleep apnea with COVID-19 pneumonia. While the COVID-19 pandemic is still evolving, understanding the correct pathophysiological mechanism of COVID-19 and ARDS is essential for appropriate treatment.

\section{References}

1. Sahu KK, Lal A, Mishra AK. An update on CT chest findings in coronavirus disease-19 (COVID-19). Heart Lung J Crit Care 2020. doi: 10.1016/j.hrtlng.2020.03.007

2. Lal A, Mishra AK, Sahu KK. CT chest findings in coronavirus disease-19 (COVID-19). J Formos Med Assoc 2020. doi: 10.1016/j.jfma.2020.03.010

3. Wu J, Liu J, Zhao X, et al. Clinical characteristics of imported cases of COVID-19 in Jiangsu province: A multicenter descriptive study. Clin Infect Dis Off Publ Infect Dis Soc Am 2020. doi: 10.1093/cid/ciaa199

4. Chen N, Zhou M, Dong X, et al. Epidemiological and clinical characteristics of 99 cases of 2019 novel coronavirus pneumonia in Wuhan, China: a descriptive study. Lancet 2020;395: 507-13.

5. Chang D, Lin M, Wei L, et al. Epidemiologic and clinical characteristics of novel coronavirus infections involving 13 patients outside Wuhan, China. JAMA 2020;323:1092-3.

6. Gattinoni L, Chiumello D, Caironi P, et al. COVID-19 pneumonia: different respiratory treatments for different phenotypes? Intensive Care Med 2020;46:1099-102.

7. Tran K, Cimon K, Severn M P, et al. Aerosol generating procedures and risk of transmission of acute respiratory infections to healthcare workers: a systematic review. PloS One 2012;7:e35797.

8. Simonds AK, Hanak A, Chatwin M, et al. Evaluation of droplet dispersion during non-invasive ventilation, oxygen therapy, nebuliser treatment and chest physiotherapy in clinical practice: implications for management of pandemic influenza and other airborne infections. Health Technol Assess Winch Eng1 2010;14:131-72.

9. Phua J, Weng L, Ling L, et al. Intensive care management of coronavirus disease 2019 (COVID-19): challenges and recommendations. Lancet Respir Med 2020;8:506-17.

10. Cheung TMT, Yam LYC, So LKY, et al. Effectiveness of noninvasive positive pressure ventilation in the treatment of acute respiratory failure in severe acute respiratory syndrome. Chest 2004; 126:845-50.

11. Sahu KK, Mishra AK, Lal A. Trajectory of the COVID-19 pandemic: chasing a moving target. Ann Transl Med 2020;8:694.

12. Marini JJ, Gattinoni L. Management of COVID-19 respiratory distress. JAMA 2020;323:2329-30.

13. Arulkumaran N, Brealey D, Howell D, Singer M. Use of noninvasive ventilation for patients with COVID-19: a cause for concern? Lancet Respir Med 2020;8:e45.

14. Robba C, Battaglini D, Ball L, et al. Distinct phenotypes require distinct respiratory management strategies in severe COVID-19. Respir Physiol Neurobiol 2020;279:103455.

15. Jain A, Doyle DJ. Stages or phenotypes? A critical look at COVID-19 pathophysiology. Intensive Care Med 2020. doi: 10.1007/s00134-020-06083-6.

16. Mishra AK, Sahu KK, Lal A, Sargent J. Patterns of heart Injury in COVID - 19 and relation to outcome [published online ahead of print, 2020 Apr 8]. J Med Virol. 2020;10.1002/ jmv. 25847 . 